\title{
A study of knowledge, attitude, practices of menstrual hygiene and its waste disposal management among adolescent schoolgirls of Gadag District of Karnataka, India
}

\author{
Anitha Dharana ${ }^{1 *}$, Sapna Kale ${ }^{2}$, Ramesh Mayappanavar ${ }^{3}$
}

\author{
${ }^{1}$ Department of Obstetrics and Gynecology, S. Nijalingappa Medical College and H. S. K. Hospital and Research \\ Hospital, Bagalkot, Karnataka, India \\ ${ }^{2}$ Consultant Obstetrician, S. Nijalingappa Medical College and H. S. K. Hospital and Research Hospital, Bagalkot, \\ Karnataka, India \\ ${ }^{3}$ Department of Community Medicine, S. Nijalingappa Medical College and H. S. K. Hospital and Research Hospital, \\ Bagalkot, Karnataka, India
}

Received: 01 October 2019

Accepted: 07 October 2019

\section{*Correspondence:}

Dr. Anitha Dharana,

E-mail: sujithbhanu1212@gmail.com

Copyright: ( $)$ the author(s), publisher and licensee Medip Academy. This is an open-access article distributed under the terms of the Creative Commons Attribution Non-Commercial License, which permits unrestricted non-commercial use, distribution, and reproduction in any medium, provided the original work is properly cited.

\begin{abstract}
Background: Adolescence is a defining time in the development of a child that is characterized by rapid physical growth and neurological sculpting, the onset of puberty and sexual maturity. Pubescent girls from developing countries face several social stigma and cultural taboos while menstruation and during menstrual hygiene management. The present study was aimed to assess the knowledge of adolescent females regarding menstruation, their perception and hygiene practice regarding menstruation.

Methods: A cross sectioned study for two months was conducted after ethical committee approval. Adolescent girl's between 10-16 years were selected from schools and data was collected by interviewing the questions in a predesigned format. Data included sanitation status of the school, knowledge about menstruation, pre-menarche, menstrual practices and beliefs, and the effect of menstruation on school life. Data was represented using percentage and simple proportions, results were drawn to arrive at conclusions of study.

Results: 1800 students were included, the mean age in the study was $13.2 \pm 1.2$ years and $12-14$ years was maximum group. $78.5 \%$ attained menarche by 13 years with 93\% Hindus. 57.5\% were unaware of knowledge and $88.6 \%$ acquired information from mother. Absenteeism from school was seen in $18.5 \%$ and $88 \%$ reported concentration problems. Dysmennorhoea was common (78\%) and $45.61 \%$ visited physician. $78.7 \%$ of the participants use sanitary pads and 55\% disposed by wrapping the pad in a newspaper and dumping in the dust bin.

Conclusions: There is good amount of awareness about menstrual hygiene and menstrual waste disposal among schoolgirls in Gadag district. Problem of menstrual waste disposal can be addressed effectively by providing and improving sanitary facilities of toilets, water and power supply through combined effort of government interventions, school managements and proper health education of girls and parents.
\end{abstract}

Keywords: Adolescence, Dysmennorhoea, Menstrual hygiene, Menstruation

\section{INTRODUCTION}

Adolescence is a defining time in the development of a child that is characterized by rapid physical growth and neurological sculpting, the onset of puberty and sexual 
maturity. WHO has defined clearly the age of adolescence as the age group of 10-19 years. Adolescent girls particularly in developing countries like India primary menstruation is considered a cultural significance and celebrated with girl as a central figure. Girls without support through puberty and during menstruation, particularly in low-resource contexts, often experience stigma and social exclusion while forgoing important educational, social and economic opportunities. Pubescent girls from developing countries face several social stigma and cultural taboos while menstruation and during "menstrual hygiene management". ${ }^{1}$

MHM is defined as use of clean menstrual material to absorb or collect blood that can be changed in privacy as often as necessary for the duration of menstruation, using soap and water for washing the body as required and having facilities to dispose of used menstrual management materials. ${ }^{2}$ Because of lack of knowledge of menstruation and menstrual hygiene girls think it as a unclean state which makes them to develop negative attitudes towards the physiological process. Unhygienic practices affect their health and cause absenteeism from regular schools and also severely impact their education. Strengthening education and health systems to provide adolescent girls with the knowledge, skills, facilities and services to manage their menstruation with dignity and free of harassment; and work on menstrual health and hygiene to support adolescent girls both in and out of school is very much required.

Hence, there is need to bring awareness about the knowledge and practice regarding menstrual hygiene among adolescent School girls and to conduct outreach camps to give knowledge and support regarding menstrual hygiene. Hence there is a compulsory need to educate girls about menstruation and healthy menstrual practices and should be a part of expanded program on health education in schools. ${ }^{3}$

The present study was aimed to assess the knowledge of adolescent females regarding menstruation, their perception and hygiene practice regarding menstruation. The study also aims to provide health education concerning the above issues.

\section{METHODS}

A school based cross sectional study was conducted by the Department of Obstetrics and Gnaecology of $\mathrm{S}$ Nijalingappa Medical College and Hospital, a tertiary care hospital. The study period was for a period of two months from June 2018 to July 2018. The study protocol was presented before the institutional ethical committee and consent was obtained. The guidelines of the ethical committee were strictly adhered during the period of the study. All the primary and secondary schools in the district of the Gadag were included and permission to visit the schools was obtained in prior from the Deputy Director of Primary Education of Gadag District. All the teachers in the school were prior informed about the details and objectives of the study. The schools were selected by random sampling. Among the schools selected all government middle and high schools after excluding boys only schools, solely residential and private schools were included in the study. Adolescent girls between 10-16 years of age were selected to maximize the likelihood of attained menarche and were aware of the information on menstrual hygiene, maintenance and waste disposal. A total of 1800 adolescent girls from various schools were selected and clearly explained about the study design, objectives and a verbal consent was obtained from all the participants of the study.

Data was obtained from all the participants by interviewing the questions prepared in a predesigned format. The format consists of fourteen questions prepared in both English and local language. Briefing was done to all the participants before the start of the study. Pre-tested structured self-administered questionnaires in the local and English languages were used to elicit information on the sanitation status of the school, knowledge about menstruation, pre-menarche, menstrual practices and beliefs, and the effect of menstruation on school life. The data was collected from senior postgraduates of the department who received intensive training on collection of the data before the commencement of the study.

\section{Statistical analysis}

The collected data was preliminarily entered into Microsoft excel spread sheet and analyzed for any corrections. Mean, median and proportions were used for analysis of the variables. Data was represented using percentage and simple proportions, results were drawn to arrive at conclusions of study and results were depicted using pie charts and tables.

\section{RESULTS}

In the present study, a total of 1800 adolescent girls who attained menarche and who fulfilled the inclusion criteria and consented for participating in the study were included. The age range of participants in the study was from 10-16 years with 10 as minimum and 16 as maximum. Majority $(37.72 \%)$ of the study participants were in the range of 12-14 years and the mean age in the study was $13.2 \pm 1.2$ years. The age of attaining menarche was 13 years in $78.5 \%$ of the study group and the mean age of attaining menarche among the girls participating in the study was 13.5 years. Majority of the girls were Hindu (93\%), Muslims (5\%) and others (2\%) (Table 1).

\section{Awareness about menstruation}

About $57.5 \%$ of the respondents were unaware and lacking knowledge about the menstruation before the first period. $88.6 \%$ of the study participants acquired primary 
information about menstruation from mothers with friends being the next reported source $9.5 \%$ and relatives $1.9 \%$. In the present study, of the 1035 students who were unaware of the menstruation till first period, $76 \%$ of the mothers were illiterate, $18 \%$ completed primary education and $6 \%$ had completed secondary education (Table 2).

Table 1: Socio demographic data of participants in the study.

\begin{tabular}{|lll|}
\hline Age group (in Years) & Number & $\%$ \\
\hline$\geq 10-\leq 12$ & 541 & 30.06 \\
\hline $12-\leq 14$ & 679 & 37.72 \\
\hline$\geq 14-16$ & 580 & 32.22 \\
\hline Religion & No & $\%$ \\
\hline Hindu & 1674 & 93 \\
\hline Muslims & 90 & 5 \\
\hline Others & 36 & 2 \\
\hline
\end{tabular}

Table 2: Details of awareness and perception.

\begin{tabular}{|lll|}
\hline A wareness & Number & $\%$ \\
\hline Yes & 765 & 42.5 \\
\hline No & 1035 & 5705 \\
\hline Source of information & & \\
\hline Mother & 1594 & 88.6 \\
\hline Friends & 172 & 9.5 \\
\hline Relatives & 34 & 1.9 \\
\hline
\end{tabular}

Table 3: Experience of first menstruation and school absenteeism among the study participants.

\begin{tabular}{|lll|}
\hline Experience & Number & $\%$ \\
\hline Fearful & 946 & 52.56 \\
\hline Embraced & 374 & 20.78 \\
\hline Confusion & 407 & 22.6 \\
\hline Excited & 73 & 4.06 \\
\hline School absenteeism & & \\
\hline Yes & 333 & 18.5 \\
\hline No & 1467 & 81.5 \\
\hline Day of absence & & \\
\hline $1^{\text {st }}$ day & 244 & 73 \\
\hline $2^{\text {nd }}$ day & 66 & 20 \\
\hline 3-5 days & 23 & 7 \\
\hline Sports participation & & \\
\hline Yes & 694 & 38.5 \\
\hline No & 725 & 40.2 \\
\hline Sometimes & 381 & 21.3 \\
\hline
\end{tabular}

\section{Perception about menstruation}

There wer $64 \%$ of the participants were aware that menstruation was a physiological process, but in about $22 \%$ of them it was the curse of God or unnatural happening which is due to passage of impurities from the body. Other reports were that it's a divine blessing for child birth. The source of bleeding was unaware in $72 \%$ of the participants with most stomach as the primary source. $22 \%$ of them reported uterus as the source of bleeding and it was due to physiological and hormonal changes in the body.

\section{Experience of first menstruation}

A $52.56 \%$ of participants expressed fear during primary menstruation followed by confusion (22.6\%), embracement $(20.78 \%)$ and excitement in $4.06 \%$ of participants (Table 3).

\section{Effect of menstruation on school experience}

In the present study, only $18.5 \%$ girls missed school during menses and maximum $81.5 \%$ attended school regularly. Of the girls who were absent during menstruation, $73 \%$ were absent on starting day of menses (day 1), 20\% for 2 days (day 1 and 2) and 7\% on 3-5 days of the period.

Majority of the students about $88 \%$ reported concentration problems even present during periods and other problems were fear of smell or getting noted by colleagues with stains on the clothes, feeling tired, unwell, dizzy and weak. $38.5 \%$ of girls participated in sports activity during menstruation, $40.2 \%$ said not participating and $21.3 \%$ informed as on and off participation depending upon the health. The proportion of girls participating (38.5\%) and not participating $(40.2 \%)$ in sports, physical activity is almost same during menses (Table 3).

\section{Health issues and clinician visit}

In the present study, $78 \%$ complained about dysmenorrhoea, $14 \%$ low backache, $22 \%$ irregular cycles and only $5 \%$ with breast pain and engorgement. In our study, $45.61 \%$ of participants reported a clinician visit during menstruation which was mostly due to dysmenorrhoea $(70.28 \%)$, followed by heavy bleeding $(23.63 \%)$ and Itch/rash in $6.09 \%$ (Table 4$)$.

\section{Menstrual hygiene management and awareness}

In our study, about $78.7 \%$ of the participants use sanitary pads during menstruation and $21.3 \%$ use absorbent cloth (Old or new). None of them had any idea of menstrual tampons and cups. The free government supply of sanitary pads has accessed more girls to use pads. Of the $78.7 \%$ of participants using sanitary pads, $70.7 \%$ replaced the pads twice a day, $28.2 \%$ once daily and $0.9 \%$ thrice daily. Maximum numbers of girls $(71.2 \%)$ change the pads twice a day and around $28.98 \%$ change once a day (Table 5).

\section{Disposal methods of sanitary pads}

Of the 1418 cases in the study, 767 (55\%) followed wrapping the pad in a newspaper and dumping in the dust bin, 524 cases $(36.95 \%)$ used to bury/ burn the pads and 
$127(8.95 \%)$ used to flush the sanitary pads in the toilet (Table 5).

Table 4: Details of health complaints and physician visit among the study participants.

\begin{tabular}{|lll|}
\hline Health complaints & & \\
\hline Dysmenorrhoea & 1403 & 78 \\
\hline Low backache & 252 & 14 \\
\hline Irregular cycles & 396 & 22 \\
\hline Breast tenderness & 90 & 5 \\
\hline Clinician visit & & \\
\hline Yes & 821 & 45.61 \\
\hline No & 658 & 36.56 \\
\hline Occasionally & 321 & 17.83 \\
\hline
\end{tabular}

Table 5: Menstrual hygiene practices and challenges.

Absorbent material used during menstruation

\begin{tabular}{|lll|}
\hline Sanitary pads & 1418 & 78.8 \\
\hline Absorbent cloth & 382 & 21.2 \\
\hline Tampoons and cups & 0 & 0 \\
\hline Frequency of pad change during & menstruation \\
\hline Once daily & 400 & 28.2 \\
\hline Twice daily & 1005 & 70.9 \\
\hline Thrice daily & 13 & 0.9 \\
\hline Disposal of absorbent material & & \\
\hline Flush in toilet & 127 & 8.95 \\
\hline Burn/Bury & 524 & 36.95 \\
\hline wrap in paper and to dustbin & 767 & 55 \\
\hline
\end{tabular}

\section{Cultural taboos and restrictions}

In our study, $78.61 \%$ of participants reported that they do not believe in social taboos. However, few reported religious restrictions, different sleeping arrangements, reduced social interactions and restricted food choices.

\section{DISCUSSION}

In the present study, 1800 participants were included from different schools and who fulfilled the inclusion criteria. The maximum age group in the study was 12-14 years. The age of attaining menarche in majority of the participants was 13 years which is similar to the findings in the studies of Tarannum $\mathrm{F}$ et al and is contrast to the findings in the study of Paria B who reported 12.8 years in his study. ${ }^{45}$ This is explained by the fact that age of menarche is influenced by various cultural, genetic, nutritional factors and most important socio economic class. Barthalakshmi et al conducted a school based cross sectional study in Chidambaram on girls from class 8th to $12^{\text {th }}$ with a mean age of menarche was $12.9 \pm 1.2$ years. $^{6}$ Majority, of the participants in the study were Hindus which doesn't have any significant impact on age of menarche. However, various studies reported differences in the age of attaining menarche which was based upon religion, place and rural or urban areas and socioeconomic considerations.
In the present study, $57.5 \%$ of respondents were unaware and lacking primary knowledge about menstruation, where as in study of Prajapati et al conducted in rural area observed that $47.5 \%$ were unaware and in study of Shabana sultan it was around $63.5 \%$ which is quite contrary to the present study. ${ }^{7,8}$ These findings are explained that the awareness is largely dependent upon the socio economic class of the participants, rural or urban settings, and literacy of the parents. In present study, $76 \%$ of mothers who were unaware of knowledge about menstruation were illiterate which supports that literacy of parents has a significant impact on knowledge of primary menstruation. $64 \%$ of participants in our study were aware that menstruation was a physiological process and findings of our study were similar to the findings of Thakur $\mathrm{H}$, who in his study in Mumbai found that $65 \%$ were unaware about the knowledge of menstruation. ${ }^{9}$ Findings of our study were in contrast to the reports of Adhikari P who reported that $78 \%$ of girls were aware of menstruation and hygienic practices. ${ }^{10}$ This is explained that awareness and practices are variable from place to place and depends on multiple factors.

In our study, $88.6 \%$ acquired primary information from mother which was similar to the findings in the studies of Mouli C V et al, but several other studies conducted at various places report mothers as a major source followed by friends, sisters and teachers as a primary source of information about menstrual knowledge. ${ }^{11}$ This variability may be due to the communication barriers existing between mother and child due to social and cultural taboos they feel awkward to discuss about it. In studies which report teachers as a primary source, the reason may be that most of the respondents may be educated till high school.

Typically, the experience of first menstruation is traumatic in most of the Indian girls. In our study, $52.56 \%$ of respondents expressed fear as the common reaction during first menstruation. Observation of our study was supported by findings of Srinivas et al who reported that fear was common in $48 \%$ of girls. ${ }^{12}$ Our study strongly suggests that girls studying in schools must be educated about menarche and menstruation and hygienic practices as a part of school curriculum. In the present study, $72 \%$ of participants reported stomach as the source of bleeding which suggest that they lack the basic knowledge of female anatomy of reproductive organs as they feel shy and uncomfortable to discuss. Similar findings were also reported by Dipali $\mathrm{N}$ et al and many other studies universally. ${ }^{13}$

In the present study, $18.5 \%$ were absent to school during menstruation and the most common reason for absenteeism was dysmenorrhoea. Our study findings were on par with the reports in the study of Ronitzsch S conducted in Germany. ${ }^{14}$ Similar findings were also reported from studies conducted in India but the range was from $16-35 \% .{ }^{15} 78.7 \%$ of participants use sanitary pads in the present study which is on par with studies 
conducted by Muralidharan A et al, who reported that $85 \%$ in his study, however few studies reported lesser rates and more in favour of absorbent cloth than sanitary pads which could be due to place of study, study population selected and social taboos existing in the study locality. ${ }^{16}$ Free provision of sanitary pads in the school was a positive thing which highlights the increase use of sanitary pads in present study. Majority $(55 \%)$ were disposing the pads by wrapping in a paper and throwing in dust bin, this highlights the awareness of use of landfills in disposal of sanitary used pads. But few studies still report a high incidence of use of absorbent cloth which may be due to restricted access to free sanitary pads and social and cultural taboos and costly sanitary napkins.

\section{CONCLUSION}

There is good amount of awareness about menstrual hygiene and menstrual waste disposal among school girls in Gadag district. Despite having free sanitary pad supply, girls are sent home, to change the pads by missing classes due to lack of proper toilet facilities and scarcity of water supply in the schools. Menstrual waste disposal is not executed properly either due to lack of water, proper toilet facilities and in some schools even though they are provided with incinerators most of them are not utilized either due to lack of power supply or they are not yet installed. These problems of menstrual waste disposal can be addressed effectively by providing and improving sanitary facilities of toilets, water and power supply through combined effort of government interventions, school managements and proper health education of girls and parents.

Funding: No funding sources Conflict of interest: None declared

Ethical approval: The study was approved by the Institutional Ethics Committee

\section{REFERENCES}

1. Jogdand K, Yerpude P. A community based study on menstrual hygiene among adolescent girls. Indian $\mathrm{J}$ Maternal Child Health. 2011;13(3):1-6.

2. United Nations. WHO/UNICEF Joint Monitoring Programme for Water Supply, Sanitation and Hygiene (JMP). UN-Water. 2012.

3. Sumpter C, Torondel B. A systematic review of the health and social effects of menstrual hygiene management. PLoS ONE. 2013;8:e62004.

4. Tarannum F, Khalique N, Eram U. A community based study on age of menarche among adolescent girls in Aligarh. Int $\mathbf{J}$ Comm Med Public Health. 2018;5:395-400.

5. Paria B, Bhattacharyya A, Das S. A comparative study on menstrual hygiene among urban and rural adolescent girls of West Bengal. J Fam Med Prim Care. 2014;3(4):413-7.

6. Barathalakshmi J, Govindarajan PK, Ethirajan N, Felix AJW. Knowledge and practice of menstrual hygiene among school going adolescent. Natl J Res Community Med. 2014;3(2):138-42.

7. Prajapati DJ, Shah JP, Khedia G. Menstrual hygiene: knowledge and practice among adolescent girls of rural Kheda. Natl J Comm Med. 2015;6(2):349-53.

8. Sultan S, Sahu DS. Knowledge, attitude and practices about menstruation and related problems in adolescent girls. Int J Reprod Contracept Obstet Gynecol. 2017;6:5235-40.

9. Thakur H, Aronnson A. Knowledge, practices, and restrictions related to menstruation among young women from low socioeconomic community in Mumbai, India. Front Public Health. 2014;2:72.

10. Adhikari P, kadel B, Dhungel SI, Mandal A. Knowledge and practices regarding menstrual hygeineamong rural aldolescent girls of Nepal. Khatmandu Univ Med J. 2007;5(3);382-36.

11. ChandraMouli V, Patel SV. Mapping the knowledge and understanding of menarche, menstrual hygiene and menstrual health among adolescent girls in lowand middle-income countries. Reprod Health. 2017;4(2):25-30.

12. Srinivas P. Perception, knowledge and practices regarding menstruation among school going girls in Karaikal. IOSR J Dent Med Sci. 2016;15(1):27-34.

13. Nemade D, Anjeneya S, Gujar R. Impact of health education on knowledge and practices about menstruation among adolescent school girl of kalomboli, Navi Mumbai. Health Populat Perspect Issues. 2009;32(4):167-75.

14. Ronitzsch S. Dropping out of school because of menstruation? An analysis of factors of success for menstrual hygiene management-projects in low and lower-middle income countries: Philipps-University Marburg, Germany; 2015.

15. Garg R, Goyal S, Gupta S. India moves towards menstrual hygiene: subsidized sanitary napkins for rural adolescent girls - Issues and challenges. Maternal Child Health J. 2012;16(4):767-74.

16. Muralidharan A, Patil H, Patnaik S. Unpacking the policy landscape for menstrual hygiene management: implications for school Wash programmes in India. Waterlines. 2015;34:79-91.

Cite this article as: Dharana A, Kale S,

Mayappanavar R. A study of knowledge, attitude, practices of menstrual hygiene and its waste disposal management among adolescent schoolgirls of Gadag District of Karnataka, India. Int J Reprod Contracept Obstet Gynecol 2019;8:4219-23. 